\title{
Dynamic Simulation, Nonlinear Control and Collision Avoid- ance of Hydraulically Driven Large Redundant Manipulators
}

\author{
Martin Schneider, Manfred Hiller and Stefan Wagner \\ Fachgebiet Mechatronik \\ Gerhard-Mercator-Universität Duisburg, Germany
}

\begin{abstract}
A detailed dynamic model of hydraulically driven mobile large-scale manipulators is presented. The model can be used as simulation model, for the calculation of the forces in the design process and for the development of the controllers, which are required for tracking given trajectories, but also to avoid vibrations. It takes into account all relevant coupling effects between hydraulic drives and arm elements as well as between flexible deformations and large nonlinear rigid body motion. The control concept makes use of the redundancy of the system in order to avoid collisions.
\end{abstract}

\section{INTRODUCTION}

The manipulators under investigation (Fig. 1) consist of three to five jib elements which are interconnected by revolute joints and which are driven by hydraulic actuators by means of transmission mechanisms (Wanner et al., 1991). They may have a total reach between 20 and 100 meters. Main areas of applications where this kind of manipulators are utilized are placing booms of truck mounted concrete pumps to transport wet concrete over large distances. In other applications in construction industry the manipulators are used as highly flexible crane systems to handle loads of about 1.500 kilograms under difficult conditions. They also serve as ramps for inspecting and cleaning constructions, e.g. high buildings or the undersides of high motorway bridges which cannot be reached from the ground.

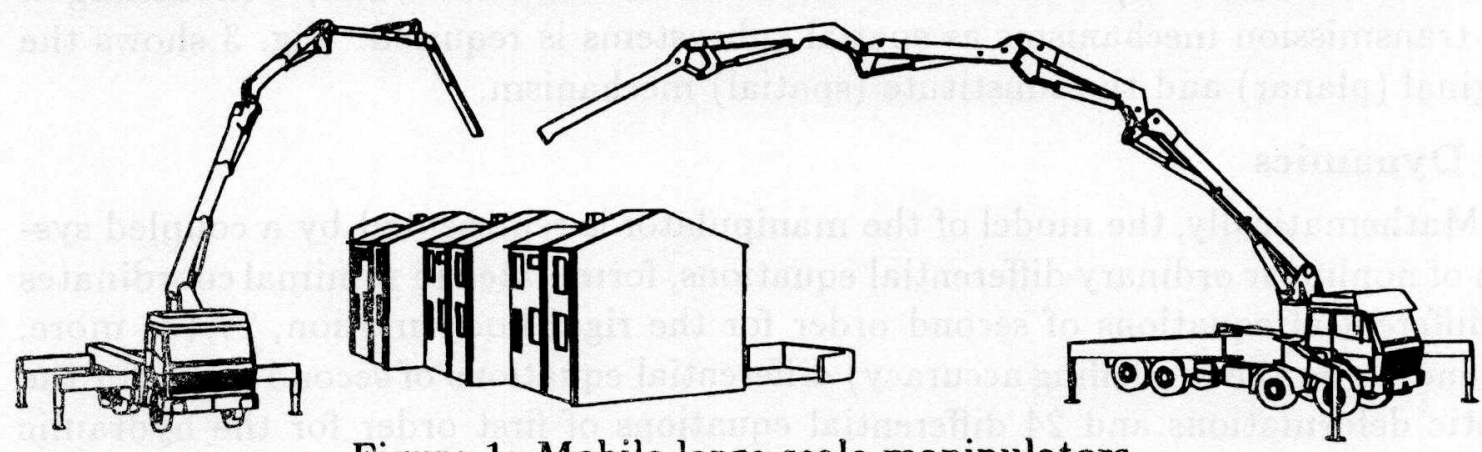

Figure 1: Mobile large-scale manipulators

As the maximum length of a single arm element is limited by the maximum total length of the truck on which the manipulator has to be transported, a long range is only feasible by a series-connection of arm elements leading to a redundant kinematical chain in the planar subsystem. As a consequence on the one hand side a complex and costly structure of the control system is required. On the other 
side, the redundant degrees of freedom provide a high mobility of the manipulator which is used for collision avoidance.

\section{MODELLING OF THE MANIPULATORS}

\subsection{Kinematical Structure}

The kinematical structure of this kind of manipulator can be regarded as a complex mechanism consisting of many rigid and flexible bodies and joints. The topology of a manipulator with five arms (right-hand side of Fig. 1) is shown in Fig. 2. The arrangement of the arm elements build on the one hand side an open kinematical chain; on the other side there appear locally closed kinematical loops $\left(L_{1}-L_{9}\right)$ due to the transmission mechanisms. In the case of backward transformation, an additional virtual joint with six degrees of freedom (three rotational, three translational) can be introduced (see Fig. 2), and an additional loop $\left(L_{10}\right)$ arises. All kinematical closed loops $\left(L_{1}-L_{10}\right)$ can be solved explicitly using the method of the characteristic pair of joints (Woernle, 1988). That holds also for kinematical loops with flexible elements.

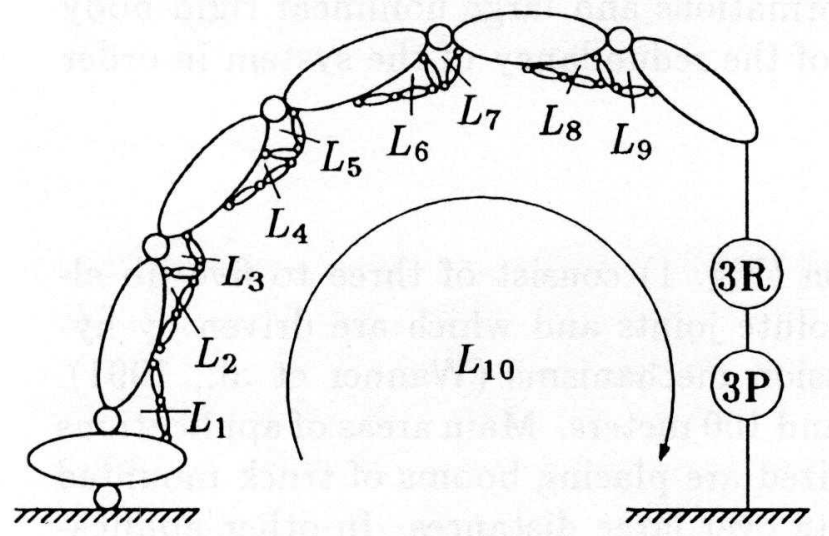

Figure 2: Topology

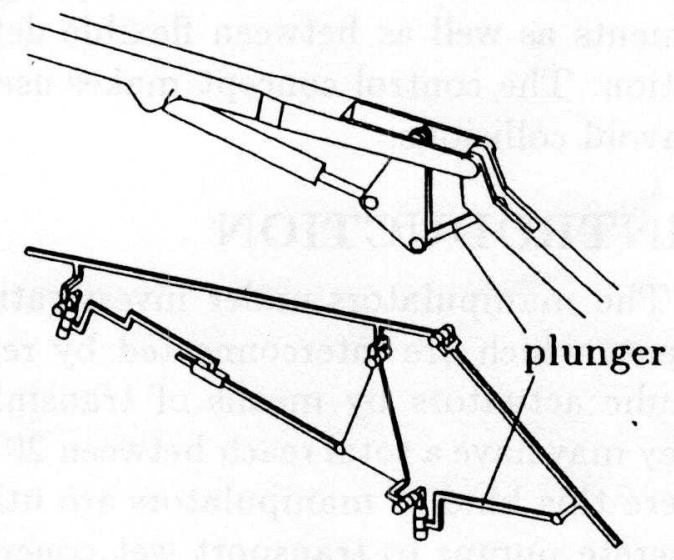

Figure 3: Transmission mechanism

Due to the deformations of the long arm elements, modelling of the manipulator as rigid body system is no longer sufficient. Taking into account bending in two planes and torsion as possible deformations of the flexible bodies, a modelling of the transmission mechanisms as spatial subsystems is required. Fig. 3 shows the original (planar) and the substitute (spatial) mechanism.

\subsection{Dynamics}

Mathematically, the model of the manipulator is represented by a coupled system of nonlinear ordinary differential equations, formulated in minimal coordinates ( 6 differential equations of second order for the rigid body motion, 27 (or more, depending on the modelling accuracy) differential equations of second order for the elastic deformations and 24 differential equations of first order for the hydraulic components). It takes into account the dynamics of the hydraulic drives including oil compressibility, the nonlinear flow and the dynamics of the servo valves, but also small deformations of the flexible arm elements and the large nonlinear rigid body 
motion. The coupling effects between the small deformations of the flexible arm elements and the large rigid body motion as well as the coupling of the hydraulic system with the mechanical system are of great importance (see section 2.5).

The modular structure of the overall system is realized by an object-oriented programming approach using the programming tool MLSBILE (Kecskeméthy, 1993), which is formulated in the language $\mathrm{C}++$. The equations of motion are computed using the kinetostatic method (residuum method), which itself makes use of the principle of D'AlEmBERT. Basic elements in this method are so-called kinetostatic transmission elements. In addition to the existing transmission elements in MISBILE those for the elastic links (section 2.3) and for the hydraulic components (section 2.4) are required.

\subsection{Elastic links}

The flexible parts of the arm elements are modelled as EULER-BERNoullibeams (Fig. 4) within the framework of a linear elasticity theory (Bremer and Pfeiffer, 1992). For the displacement vector $u=[u, v, w]^{T}$ and the vector of the Bryant-angles $\varphi=[\alpha, \beta, \gamma]^{T}$ the Ritz approaches $v(x, t)=\boldsymbol{\Phi}_{v}^{T}(x:) \boldsymbol{q}_{v}(t)$, $w(x, t)=\boldsymbol{\Phi}_{w}^{T}(x) \boldsymbol{q}_{w}(t), \alpha(x, t)=\boldsymbol{\Phi}_{\alpha}^{T}(x) \boldsymbol{q}_{\alpha}(t)$ and inner constraints for $u, \beta$ and $\gamma$ are assumed. The elastic degrees of freedom are collected in the vector $\boldsymbol{q}_{e}=\left[\boldsymbol{q}_{v}, \boldsymbol{q}_{w}, \boldsymbol{q}_{\alpha}\right]^{T}$.

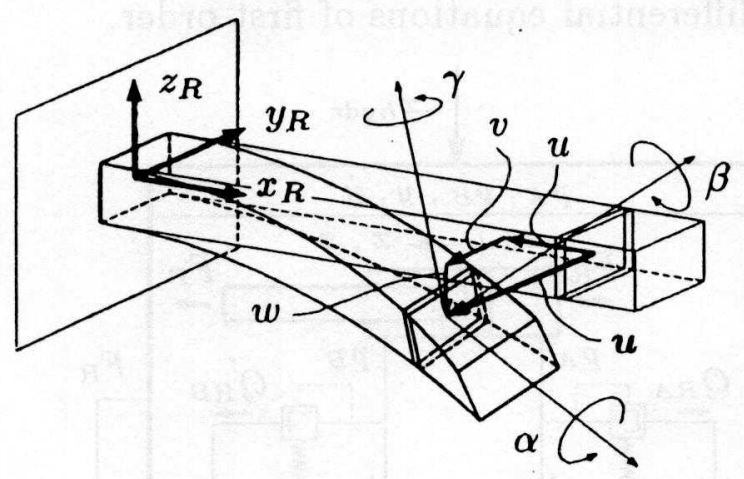

Figure 4: Euler-Bernoulli-beam

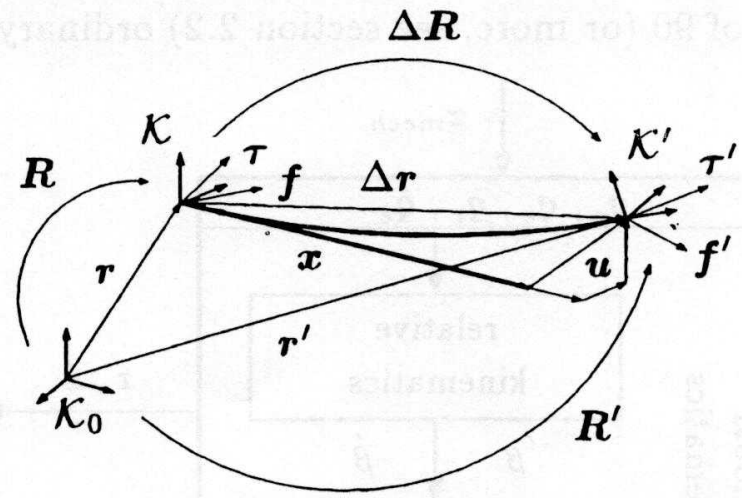

Figure 5: Kinetostatic quantities

The following relations describe the transmission of orientation (1), position (2), velocity (3) and acceleration (4) from an input frame $\mathcal{K}$ to an output frame $\mathcal{K}^{\prime}$, while (5) describes the force transmission in inverse direction (see Fig. 5). The vector $\boldsymbol{Q}_{e}$ in $\mathrm{Eq}$. (5) denotes the generalized forces of the generalized elastic coordinates due to the acting forces and torques. For more details refer to Schneider and Hiller, 1995.

$$
\begin{gathered}
\boldsymbol{R}^{\prime}=\boldsymbol{R} \cdot \Delta \boldsymbol{R} ; \quad \Delta \boldsymbol{R} \approx \boldsymbol{I}+\tilde{\varphi} \\
\boldsymbol{r}^{\prime}=\Delta \boldsymbol{R}^{T} \boldsymbol{r}+\Delta \boldsymbol{r} ; \quad \Delta \boldsymbol{r}=\Delta \boldsymbol{R}^{T}(\boldsymbol{x}+\boldsymbol{u}) \\
{\left[\boldsymbol{\omega}^{\prime}, \boldsymbol{v}^{\prime}\right]^{T}=J_{\phi}\left[\boldsymbol{\omega}, \boldsymbol{v}, \dot{\boldsymbol{q}}_{e}\right]^{T}} \\
{\left[\dot{\boldsymbol{\omega}}^{\prime}, \boldsymbol{a}^{\prime}\right]^{T}=J_{\phi}\left[\dot{\boldsymbol{\omega}}, \boldsymbol{a}, \ddot{\boldsymbol{q}}_{e}\right]^{T}+\left[\boldsymbol{\epsilon}_{\boldsymbol{\omega}}, \boldsymbol{\epsilon}_{a}\right]^{T}}
\end{gathered}
$$




$$
\left[\tau, f, Q_{e}\right]^{T}=J_{\phi}^{T}\left[\tau^{\prime}, f^{\prime}\right]^{T}
$$

The generalized forces arising from the elastic potential and the mass properties are treated as so-called leaf elements. On closer examination of the Jacobian $\boldsymbol{J}_{\phi}$ it can be seen that there is a strong coupling between flexible deformations and rigid body motion.

\subsection{Hydraulic components}

The modelling of hydraulic components is described in detail in the literature for oil hydraulics. For the use within modelling of multibody systems, some adaptions have to be done. Here, a hydraulic cylinder, a servo valve and two relief valves are combined to a hydraulic drive (see right part of Fig. 6). The transmission element for the hydraulic drive is treated as a force element, but in contrast to passive force elements (e.g. springs and dampers) the hydraulic drive has its own state variables $p_{A}, p_{B}, y$ and $\dot{y}$.

\subsection{Coupling of mechanics and hydraulics}

By modelling the hydraulic components in the manner described above, the coupling of mechanics and hydraulics is given in a natural way, see Fig. 6. Eq. (7) shows the overall system of strongly coupled (stiff) differential equations, consisting of 90 (or more, see section 2.2) ordinary differential equations of first order.

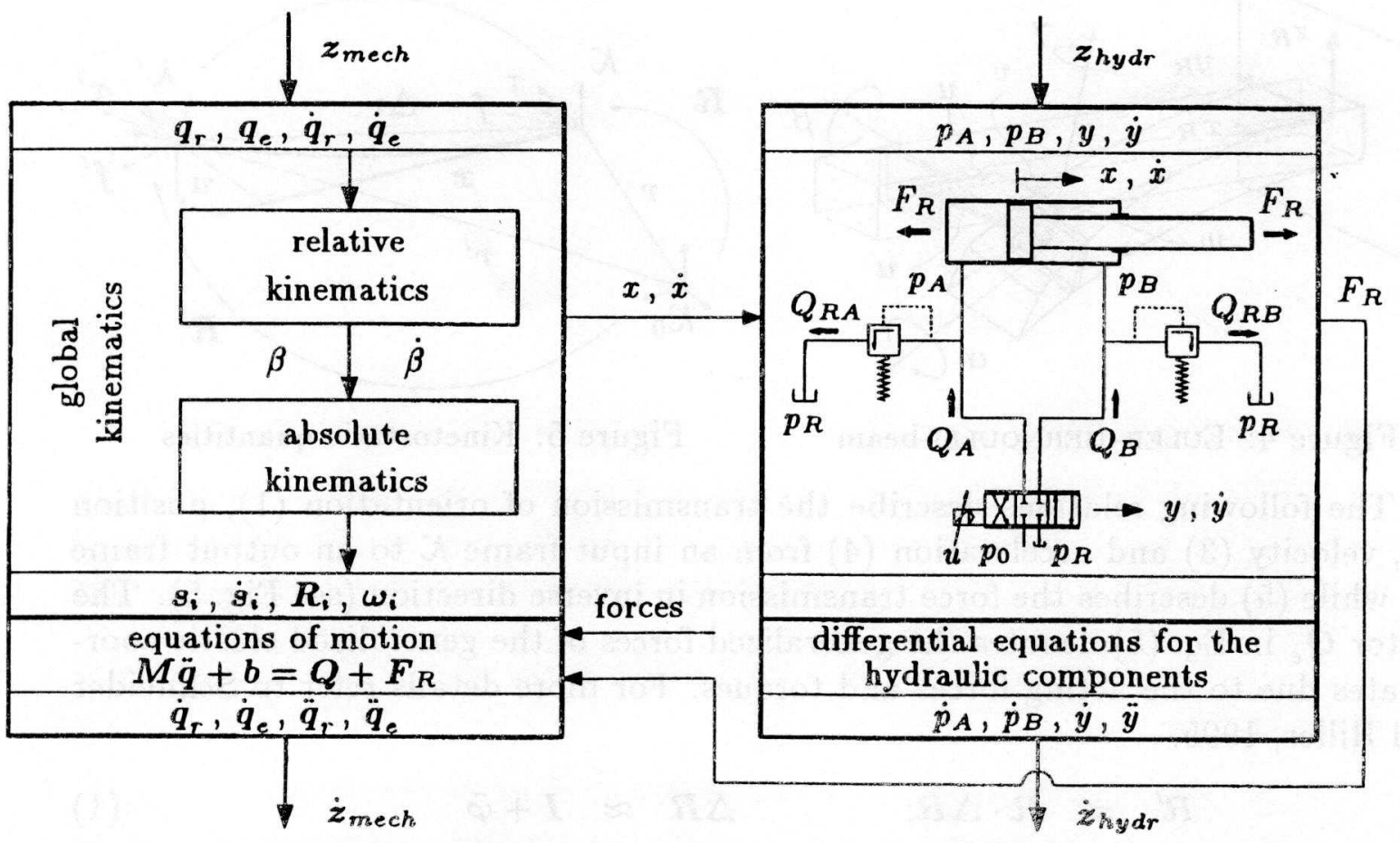

Figure 6: Coupling of mechanics und hydraulics

$$
\boldsymbol{q}=\left[\boldsymbol{q}_{r}, \boldsymbol{q}_{e}\right]^{T} ; \quad \boldsymbol{z}_{\text {mech }}=[\boldsymbol{q}, \dot{\boldsymbol{q}}]^{T} ; \quad \boldsymbol{z}=\left[\boldsymbol{z}_{\text {mech }}, \boldsymbol{z}_{h y d r}\right]^{T}
$$




$$
\dot{z}=\left[\begin{array}{c}
\dot{z}_{m e c h} \\
\dot{z}_{h y d r}
\end{array}\right]=\left[\begin{array}{c}
\dot{\boldsymbol{q}} \\
\boldsymbol{M}^{-1}(\boldsymbol{q})\left[\boldsymbol{b}(\boldsymbol{q}, \dot{\boldsymbol{q}})-\boldsymbol{Q}(\boldsymbol{q}, \dot{\boldsymbol{q}})-\boldsymbol{F}_{R}\left(\boldsymbol{q}, \dot{\boldsymbol{q}}, \boldsymbol{p}_{A}, \boldsymbol{p}_{B}\right)\right] \\
\dot{\boldsymbol{p}}_{A}\left(\boldsymbol{q}, \dot{\boldsymbol{q}}, \boldsymbol{p}_{A}, \boldsymbol{p}_{B}, \boldsymbol{y}\right) \\
\dot{\boldsymbol{p}}_{B}\left(\boldsymbol{q}, \dot{\boldsymbol{q}}, \boldsymbol{p}_{A}, \boldsymbol{p}_{B}, \boldsymbol{y}\right) \\
\dot{\boldsymbol{y}} \\
\ddot{\boldsymbol{y}}(\boldsymbol{y}, \dot{\boldsymbol{y}}, \boldsymbol{u})
\end{array}\right]
$$

\section{COMPARISON OF STATIC AND DYNAMIC FORCES}

During the design process, one needs information about the forces and torques acting in the individual elements of the construction. Due to the complexity of the whole system, it is even difficult to determine the static forces by static models. But it is impossible to calculate the dynamic forces of a moving system - e.g. the forces in the hydraulic drives - by a purely statical approach. In addition, if force control is used in the control concept, the dynamic forces are required for computing the controller output.

The model described in section 2 provides these forces. Some comparisons have been done to show the differences between static and dynamic forces. The simulations in section 3.1 and 3.2 refer to the manipulator with four arms (lefthand side of Fig. 1). The curves for the static forces result from the assumption that there is static equilibrium.

\subsection{Unfolding}

The following figures show the comparisons during an unfolding process of the manipulator. Fig. 7 represents the shearing force $F_{y}$ at the revolute joint between the first and the the second arm, and Fig. 8 shows the compressive force $F_{x}$ in "the plunger of the transmission mechanism (see Fig. 3) at that joint.

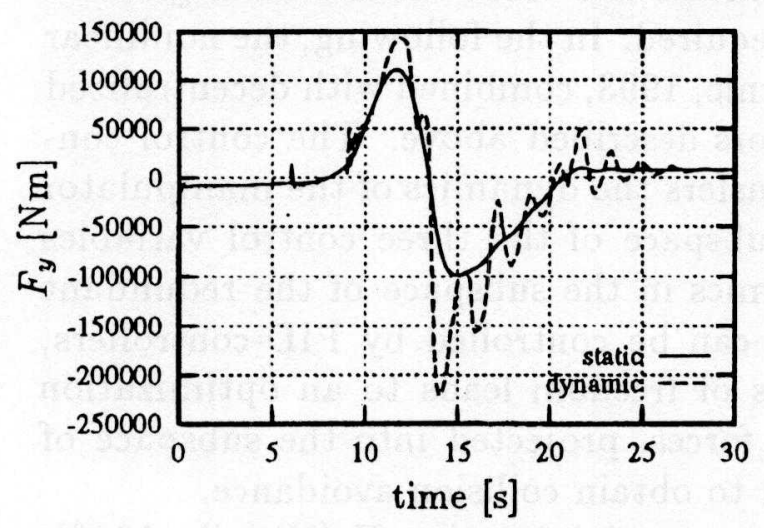

Figure 7: Shearing force

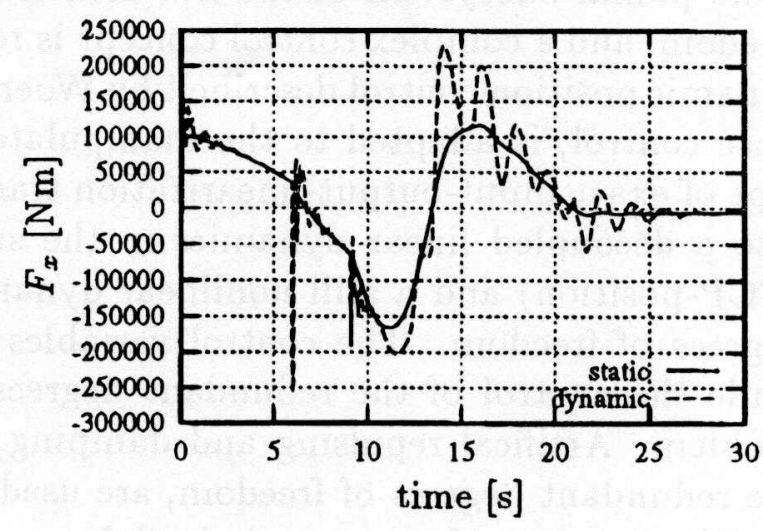

Figure 8: Compressive force

\subsection{Pumping wet concrete}

In the case of pumping wet concrete in a pulsating mode vibrations with large amplitudes (up to $0.5 \mathrm{~m}$ and more at the free end of the pumping device) can be observed which are very dangerous for the operating crew. One aim of the control concept is to avoid these vibrations. Fig. 9 shows the vertical deflection 
of the TCP (tool center point) during the pump process, where the manipulator holds the position represented in the upper left corner of Fig. 9 . It can be seen that modelling of the arm elements as flexible beams on the one hand side and rigid bodies on the other side leads to different results. Fig. 10 shows again the compressive force in the plunger.

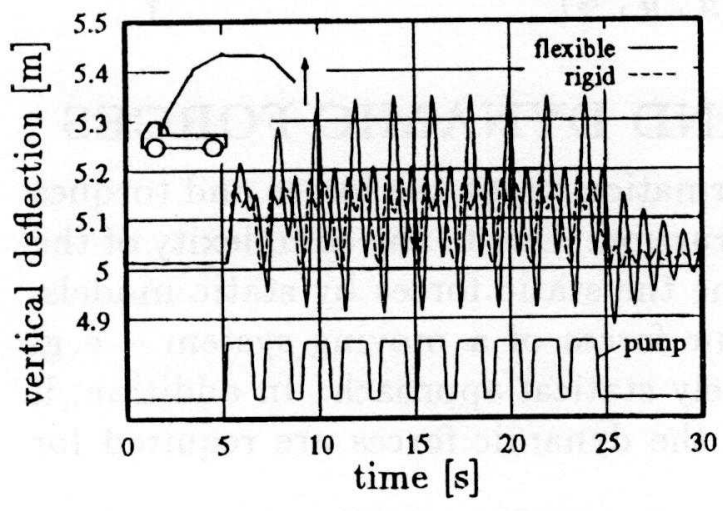

Figure 9: Vertical deflection

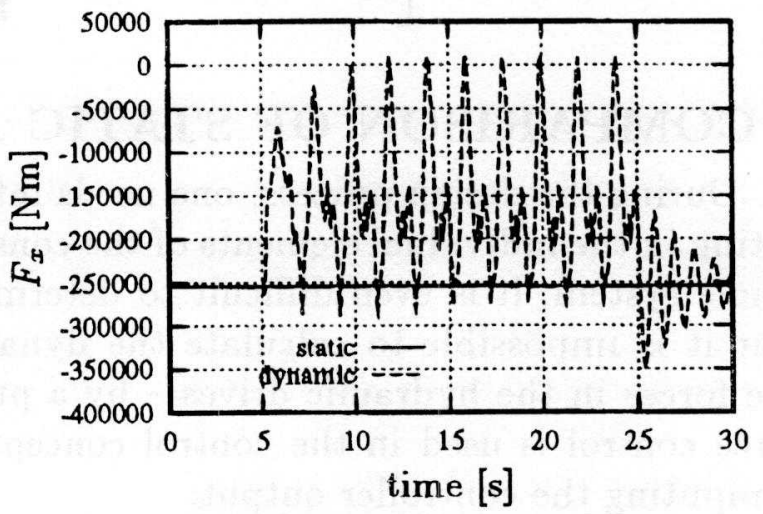

Figure 10: Compressive force

\section{OBSTACLE AVOIDANCE}

\subsection{Input-output linearization}

In Euclidean space, the position of the TCP is determined by six parameters: three for the translation and three for the rotation. Here, only the three translational coordinates are considered, since the orientation of the TCP is either insignificant (e.g. pumping concrete) or can be controlled by additional joints of an attached working platform. Therefore - considering a manipulator with five arms - the planar subsystem of the five arm elements has three redundant degrees of freedom, and a complex control concept is required. In the following, the nonlinear dynamic position control described by Woernle, 1993, combined with decentralized force control, is adapted to the manipulators described above. The control concept of exact input-output-linearization transfers the dynamics of the manipulator into a decoupled linear dynamics in the subspace of the three control variables (TCP-position) and a still nonlinear dynamics in the subspace of the redundant degrees of freedom. The control variables can be controlled by PID-controllers, while the control of the redundant degrees of freedom leads to an optimization problem. Artifical repulsing and damping forces, projected into the subspace of the redundant degrees of freedom, are used to obtain collision avoidance.

The repulsing forces are derived from a potential function II (Khatib, 1986), which has a repulsing influence on the manipulator arm, and depend on the shortest distance $d\left(\boldsymbol{r}_{i}\right)$ between the obstacle and a discrete point (absolute position vector $r_{i}$ ), see Fig. 11. The number of discrete points must be adapted to the dimension of the obstacles: the smaller an obstacle, the larger the number of required discrete points. 


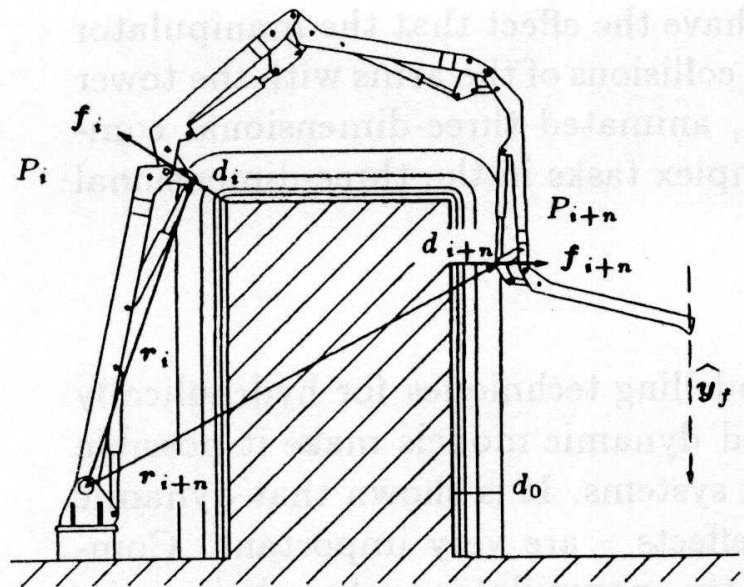

Figure 11: Repulsing forces

A simulation environment with several spatial obstacles has been created to test the control concept (Wagner, 1994). In a first step a simplified model of the manipulator is regarded by treating all bodies as rigid and the hydraulic drives as ideal actuators, thus supplying the forces required by the controllers. Currently the simulation of the complete system with flexible arm elements and nonlinear hydraulic components is under investigation

\subsection{Obstacles}

For testing, several objects from an outdoor area are chosen as obstacles, e.g. buildings, vegetation, lighting systems and pipelines. They are replaced by mathematical simple envelopes (sphere, cylinder and rectangular parallelepiped), so that the simple distance functions point-to-point, point-to-line and point-to-plane can be used to calculate the shortest distance between a discrete point and an obstacle. In Fig. 12 these envelopes are represented. Outside an outer envelope (distance $d_{0}$ from the obstacle) all repulsing forces are zero.
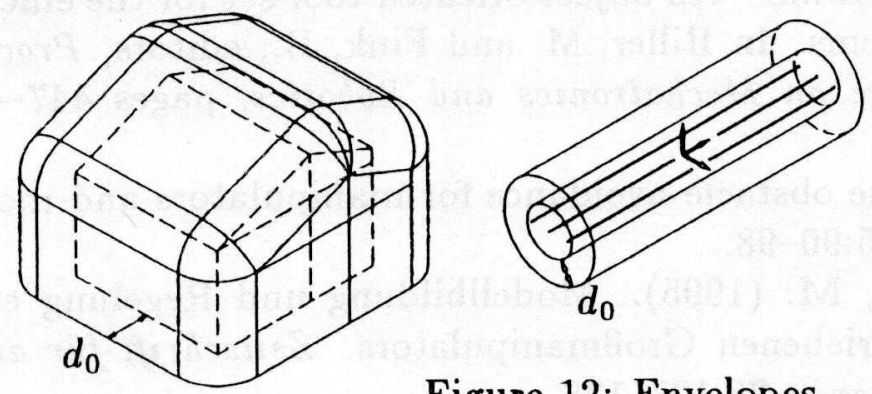

Figure 12: Envelopes

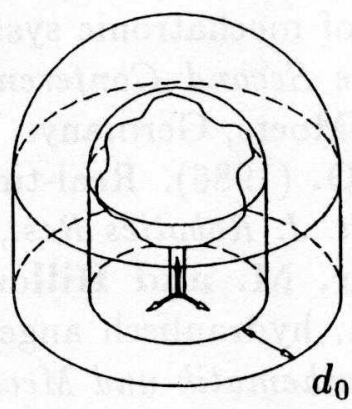

\subsection{Examples}

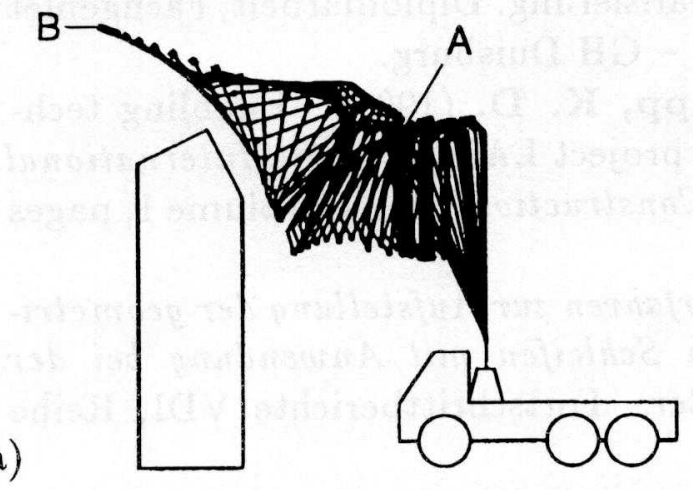

b)

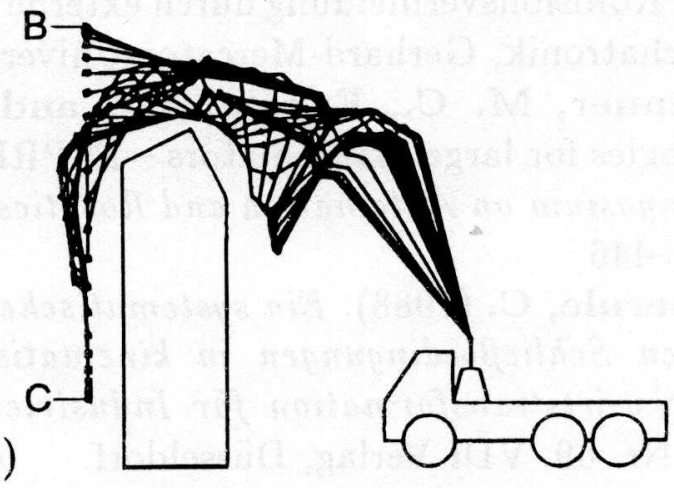

Figure 13: Obstacle avoidance

Fig. 13 shows a simulation result where the manipulator has the task to track a given trajectory (a) straight line from $\mathrm{A}$ to $\mathrm{B}, \mathrm{b}$ ) straight line behind the tower: 
from $\mathrm{B}$ to $\mathrm{C})$. The repulsing forces of the roof have the effect that the manipulator arms snuggle up to the contour of the roof, and collisions of the arms with the tower contour are completely avoided. In addition, animated three-dimensional computer simulations can be used to visualize complex tasks in the three-dimensional workspace.

\section{CONCLUSION}

This paper gives a survey of nonlinear modelling techniques for hydraulically driven large-scale manipulators. The presented dynamic models make it possible to understand the behaviour of these complex systems. It is shown that dynamic effects - and here in particular the coupling effects - are very important. Comparing the forces in the individual elements of the manipulator makes obvious the difference between static and dynamic formulations. The dynamic models are used as simulation models to replace the real system in the developmental stage, but also as a basis for nonlinear control concepts. With recent advances in the capabilities of computers, such complex control concepts will be increasingly applied to support the operator of the manipulator.

\section{REFERENCES}

Bremer, H. and Pfeiffer, F. (1992). Elastische Mehrkörpersysteme. Teubner Studienbücher Mechanik. B. G. Teubner, Stuttgart.

Kecskeméthy, A. (1993). MовILE - An object-oriented tool-set for the efficient modeling of mechatronic systems. In Hiller, M. and Fink, B., editors, Proceedings of the Second Conference on Mechatronics and Robotics, pages 447-462, Duisburg/Moers, Germany.

Khatib, O. (1986). Real-time obstacle avoidance for manipulators and mobile robots. Int. J. Robotics Res., 5:90-98.

Schneider, M. and Hiller, M. (1995). Modellbildung und Regelung eines elastischen, hydraulisch angetriebenen Großmanipulators. Zeitschrift für angewandte Mathematik und Mechanik, 75:127-128.

Wagner, S. (1994). Nichtlineare Lageregelung eines redundanten Manipulators mit Kollisionsvermeidung durch externe Linearisierung. Diplomarbeit, Fachgebiet Mechatronik, Gerhard-Mercator-Universität - GH Duisburg.

Wanner, M. C., Engeln, W., and Rupp, K. D. (1991). Enabling technologies for large manipulators - ESPRIT II project LAMA. In 8th International Symposium on Automation and Robotics in Construction ISARC, volume 1, pages 439-446.

Woernle, C. (1988). Ein systematisches Verfahren zur Aufstellung der geometrischen Schließbedingungen in kinematischen Schleifen mit Anwendung bei der Rückwärtstransformation für Industrieroboter. Fortschrittberichte VDI, Reihe 18, Nr. 59. VDI Verlag, Düsseldorf.

Woernle, C. (1993). Nonlinear control of constrained redundant manipulators. In Angeles, J., Hommel, G., and Kovacs, P., editors, Computational Kinematics, pages 119-128. Kluwer Academic Publishers. 